\title{
The Class of Groups All of Whose Subgroups with Lesser Number of Generators are Free is Generic
}

\author{
G. N. Arzhantseva and A. Yu. Ol'shanskii
}

UDC 512.5

\begin{abstract}
It is shown that, in a certain statistical sense, in almost every group with $m$ generators and $n$ relations (with $\boldsymbol{m}$ and $n$ chosen), any subgroup generated by less than $\boldsymbol{m}$ elements (which need not belong to the system of generators of the whole group) is free. In particular, this solves Problem 11.75 from the Kourov Notebook. In the proof we introduce a new assumption on the defining relations stated in terms of finite marked groups.
\end{abstract}

\section{Introduction}

As is well known, in the fundamental group of an oriented closed compact surface of genus $g$, all subgroups of infinite index are free. In particular, this means that in the group

$$
S_{g}=\left\langle a_{1}, \ldots, a_{g}, b_{1}, \ldots, b_{g} \mid\left[a_{1}, b_{1}\right] \ldots\left[a_{g}, b_{g}\right]=1\right\rangle
$$

with $2 g$ generators, any subgroup with lesser number of generators (not necessarily from the original set $\left.\left\{a_{1}, \ldots, a_{g}, b_{1}, \ldots, b_{g}\right\}\right)$ is free. In this connection the second author posed the problem of finding conditions on the defining relations under which the subgroups of a finitely defined group with a "small" number of generators are free. Such conditions were found by V. S. Guba in the paper [1], where subgroups with two generators were considered. Besides the condition of small cancellation, there was a condition on "long 2-subwords" of defining words.

To study groups with free $k$-generated subgroups with $k>2$, we had to find an appropriate generalization of the latter condition, and this seemed to be completely unclear. Therefore, in [1] (and then in $[2,11.75])$ we posed the problem of whether the class of $m$-generated groups all of whose $(m-1)$ generated subgroups are free is "generic". This problem is solved in the present note.

For some chosen numbers $m$ and $n$ of generators and defining relations, respectively, a subclass $C$ of finite presentations is said to be generic if almost every group with $m$ generators and $n$ relations belongs to $C$ provided that the defining relations are sufficiently long. The study of generic properties is useful at least because the combination of an arbitrary finite number of generic properties is again a generic property of groups.

To state our assertions more exactly, consider a group alphabet $X_{m}=\left\{x_{1}^{ \pm 1}, \ldots, x_{m}^{ \pm 1}\right\}$, where $m>1$, and a set of cyclically reduced words $\left\{r_{1}, \ldots, r_{n}\right\}$ in this alphabet of length $\left|r_{i}\right| \leqslant t$. Denote by $N_{m, n, t}$ the number of all presentations of groups of the form

$$
G=\left\langle x_{1}, \ldots, x_{m} \mid r_{1}=1, \ldots, r_{n}=1\right\rangle
$$

satisfying this condition and denote by $N_{m, n, t}^{f}$ the number of presentations such that any $(m-1)$ generated subgroup of $G$ is free (and, moreover, $G$ really contains free subgroups of an arbitrary finite rank).

Theorem 1. There is a number $c=c(m, n)>0$ such that the ratio $N_{m, n, t}^{f} / N_{m, n, t}$ is greater than $1-\exp (-c t)$.

As a consequence, we obtain the answer to questions in [1] and [2, 11.75]. Namely, denote by $N_{d}=$ $N_{d}(m, n)$ the number of all presentations of groups of the form (1) such that $\left|r_{1}\right|+\cdots+\left|r_{n}\right|=d$, where $r_{z}$ is a cyclically reduced word in the alphabet $X_{m}$ and $N_{d}^{f}$ is the number of such presentations for which any $(m-1)$-generated subgroup of $G$ is free.

Translated from Matematicheskie Zametki, Vol. 59, No. 4, pp. 489-496, April, 1996

Original article submitted January 5, 1995. 
Theorem 2. For every $m>1$ and for any chosen $n$ we have

$$
\lim _{d \rightarrow \infty} \frac{N_{d}^{f}}{N_{d}}=1 .
$$

When solving the main problem, we use marked graphs as an auxiliary tool. For example, it is clear that any word in the variables $x_{1}^{ \pm 1}, x_{2}^{ \pm 1}, x_{3}^{ \pm 1}$ can be read when going around the union of three cycles of length 1 shown in Fig. 1.

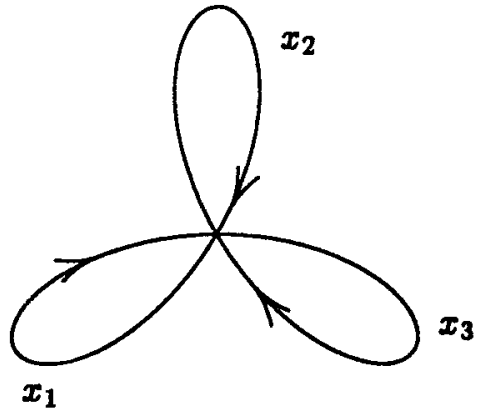

Fig. 1.

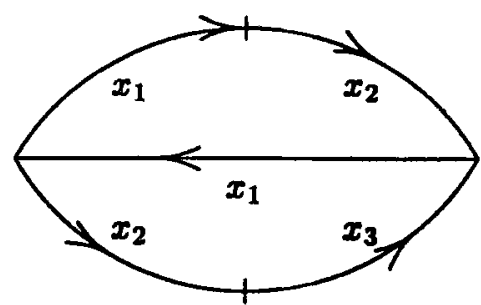

FIG. 2.

As far as the graph in Fig. 2 is concerned, we can read words as long as desired when going around this graph, for example:

$$
x_{1} x_{2} x_{1} x_{2} x_{3} x_{2}^{-1} x_{1}^{-1} x_{2} x_{3} x_{1} \ldots,
$$

and the number of these words increases exponentially together with their length, but at the same time the fraction of all uncancellable words of length $\ell$ among all words of length $\ell$ in three variables exponentially decreases as $\ell$ increases. (The words $x_{3}^{2}$ and $x_{1} x_{2} x_{1}^{-1}$ are already unreadable along this graph.)

\section{§1. Marked graphs and subgroups}

Let $\Gamma$ be a connected marked graph, that is, a finite connected 1-complex such that to each of its free oriented edges $e$ a mark $\varphi(e)=x$ with $x \in X_{m}$ is assigned, and we have $\varphi\left(e^{-1}\right)=\varphi(e)^{-1}$. One of the vertices, say, $o$, is regarded as a labeled vertex in $\Gamma$. Clearly, all words starting and ending at $o$ that can be read on the cycles of the graph $\Gamma$ form a subgroup $H=H(\Gamma)$ of the free group $F_{m}=F\left(X_{m}\right)$ with basis $X_{m}$ (although an element of $H$ can be represented by different cycles, due to possible cancellations).

Clearly, $H(\Gamma)$ is a homomorphic image of the fundamental group $\pi_{1}(\Gamma)$ under the homomorphism induced by the marking function $\varphi$. It is also clear that each finitely generated subgroup $H \subset F_{m}$ can be presented in this way. It suffices to choose in $H$ a system of generators $\left\{w_{1}, w_{2}, \ldots\right\}$ and to identify the starting vertices of each of simple cycles with corresponding marks $w_{1}, w_{2}, \ldots$. (Here by the mark $\varphi(p)$ of a simplicial path $p=e_{1} \ldots e_{n}$ we mean the word $\varphi\left(e_{1}\right) \ldots \varphi\left(e_{n}\right)$.) For the usefulness of this way of defining a subgroup of a free group, see, for example, [3].

If $G$ is an m-generated group given by the presentation (1), then each its subgroup $H$ can also be given by some graph $\Gamma$, namely, it suffices to consider the preimage of this group under the natural homomorphism $F_{m} \rightarrow G$.

A marked connected graph is said to be reduced if $\Gamma$ has no vertex with two outgoing edges with the same marks, and there are no vertices of valency 1 , except possibly for the labeled vertex $o$. Every graph $\Gamma$ defining a subgroup $H$ can be replaced by a reduced graph [3] by means of transformations of the first kind (pasting together a pair of edges with the same starting point and equal marks) and of the second kind (deleting a vertex of valency one together with the incoming edge).

Everywhere below, by an arc we mean a path $p$ all of whose vertices (possibly except for the starting point $p_{-}$and the endpoint $p_{+}$) differ from the labeled vertex 0 and are of valency 2 .

For a graph $\Gamma$ defining a subgroup $H$ of the group $G$ with the presentation (1), we define a transformation of the third kind as well:

Adding an arc. Let vertices $o_{1}$ and $o_{2}$ in $\Gamma$ be joined by a path $p$ such that $\varphi(p) \equiv w$ and the word $w$ is equal in $G$ to some word $v$. Then let us add to $\Gamma$ a new graph formed by a single arc $q$ with mark 
$\varphi(q) \equiv v$ such that $o_{1}=q_{-}$and $o_{2}=q_{+}$(the starting point and the endpoint of the path $q$ ) are the only common points of the new graph and $\Gamma$.

Inverse transform. Let $q$ be an arc in $\Gamma$, let $q_{-}=o_{1}$ and $q_{+}=o_{2}$, and suppose the vertices $o_{1}$ and $\mathrm{O}_{2}$ can be joined in $\Gamma$ by another path $p$ such that $p$ and $q$ have no nonoriented edges in common and $\varphi(p)=\varphi(q)$ in the group $G$. Then we can delete all edges and vertices of the path $q$, except $o_{1}$ and $o_{2}$, from $\Gamma$.

Lemma 1. If the graph $\Gamma^{\prime}$ is obtained from the graph $\Gamma$ by means of transformations of the first, second, and third kind (or their inverses), then this graph defines the same subgroup of the group $G$ as $\Gamma$ does.

The assertion is established by a clear item-by-item examination of possible types of transformations.

\section{§2. $\mu$-readable words}

Let $m \geqslant 2$. A nonempty uncancellable word $w$ of length $\ell$ in the alphabet $X_{m}=\left\{x_{1}^{ \pm 1}, \ldots, x_{m}^{ \pm 1}\right\}$ is said to be $\mu$-readable $\left(\right.$ or $(\mu, m)$-readable) for some $\mu$, where $0<\mu \leqslant 1$, if there exists a $X_{m}$-marked reduced graph $\Gamma$ with the following properties:

(a) the number of the edges of this graph is at most $\mu \ell$;

(b) the Euler characteristic of the graph $\Gamma$ exceeds $1-m$ (in other words, $\pi_{1}(\Gamma)$ is of rank less than $m$, that is, $\Gamma$ has less than $m$ independent cycles);

(c) the word $w$ can be read along some path of the graph $\Gamma$.

Without the second condition, the definition becomes meaningless, because every word in $m$ variables can be read along the union of $m$ circles of length 1 .

Let us estimate the number of $\mu$-readable words of length $\ell$. Consider the graph from the definition of $\mu$-readable words. Since it has at most one vertex of valency one and its Euler characteristic exceeds $1-m$, the number of vertices of valency $\geqslant 3$ is at most $2 m-3$. By the same argument, the number of maximal arcs in $\Gamma$ is at most $3 m-4$. Therefore, the number of topological types of graphs is bounded by a constant $C(m)$.

The number of possible decompositions of simple arcs of the graph of a certain type into $\leqslant \mu \ell$ edges has an upper bound of the form

$$
\sum_{1 \leqslant k \leqslant[\mu \ell]}\left(\begin{array}{c}
3 m+k-5 \\
k-1
\end{array}\right) \leqslant(\mu \ell)^{3(m-1)} .
$$

To each of $\leqslant \mu \ell$ edges we assign one of $2 m$ letters, and this can be done by at most $(2 m)^{\mu \ell}$ ways. Thus, the number of marked graphs from the definition of a $\mu$-readable word is less than

$$
C(m)(\mu \ell)^{3(m-1)}(2 m)^{\mu \ell} \text {. }
$$

Now we estimate the number of uncancellable words of length $\ell$ that are readable on the given graph $\Gamma$ starting from an arbitrary vertex. The initial edge can be chosen by $\leqslant 2 \mu \ell$ ways. After passing each edge, there are $\leqslant 2 m-2$ ways to continue the path, because the valency of each vertex is at most $2 m-1$ (this follows from the restriction on the Euler characteristic and from the fact that there is at most one vertex of valency 1). Hence, on $\Gamma$ we can read at most $2 \mu \ell(2 m-2)^{\ell-1}$ words of length $\ell$.

Together with (2), we can conclude that the number of $\mu$-readable words of length $\ell$ is at most

$$
2 C(m)(\mu \ell)^{3 m-2}(2 m)^{\mu \ell}(2 m-2)^{\ell-1} .
$$

Now we assume that

$$
\mu<\log _{2 m}\left(1+\frac{1}{4(m-1)}\right) .
$$

Then (3) implies the following assertion.

Lemma 2. Under condition (4), we can indicate a constant $k=k(\mu, m)$ such that the number of $\mu$-readable words of length $\ell$ in the alphabet $X_{m}$ is less than $k(2 m-3 / 2)^{\ell}$. 


\section{§3. Condition for defining words}

In this section we consider a $(\lambda, \mu)$-condition on the system of defining words. Here we choose $\lambda>0$ and a positive $\mu$ satisfying condition (4).

Recall (see also [4]) that the condition of small cancellation $C^{\prime}(\lambda)$ for the presentation (1) means that if $R$ denotes the set of all cyclic permutations of the words $\left\{r_{1}, \ldots, r_{n}\right\}$ and their inverses, then for different $r, r^{\prime} \in R$, the length of common beginning of the words $r$ and $r^{\prime}$ is less than $\lambda \min \left(|r|,\left|r^{\prime}\right|\right)$.

Lemma 3. Among all finite sequences $\left\{r_{1}, \ldots, r_{n}\right\}$ of cyclically uncancellable words whose lengths are at most $t$, the fraction of sequences satisfying condition $C^{\prime}(\lambda)$ is not less than $1-a(2 m-1)^{-\lambda t / 5}$ for $t \geq a$, where $a=a(m, n, \lambda)>0$.

Proof. Clearly, the number $N_{t, m}$ of cyclically uncancellable words of length $\leq t$ is not less than $(2 m-1)^{t-1}$; hence, the number of $n$-term sequences $N_{t, m, n}$ composed of these words is at least

$$
(2 m-1)^{n(t-1)} \text {. }
$$

Now consider a cyclically reduced word $r$ such that $t / 2<|r| \leq t$ and assume that the system does not satisfy condition $C^{\prime}(\lambda)$. It is readily seen that in this case the cyclic word $r$ contains two disjoint subwords $u$ and $v$ such that $u=v^{ \pm 1}$ and $|u| \geq \lambda t / 4$. However, the number of all words satisfying this restriction is less than $3 t^{2}(2 m-1)^{t-\lambda t / 4}$. Hence, the number of sequences $\left\{r_{1}, \ldots, r_{n}\right\}$ of words of length $\leq t$ that contain such a word $r$ is less than $3 n t^{2}(2 m-1)^{t n-\lambda t / 4}$. Similarly, we can find an upper bound for the number of sequences $\left\{r_{1}, \ldots, r_{n}\right\}$ composed of cyclically reduced words with length in the interval $(t / 2, t]$ such that for some $i \neq j$ the words $r_{i}$ and $r_{j}$ have a common subword of length $\geq \lambda t / 2$. It suffices to take the estimate $3 t^{2}(2 m-1)^{t n-\lambda t / 2}$. Finally, the number of words of length $\leq t / 2$ is at most $2 m(2 m-1)^{t / 2}$. Therefore, the number of sequences of length $n$ that contain such words is less than $2 m n(2 m-1)^{t(n-1)+t / 2}$. Adding the obtained bounds and dividing the sum by $N_{t, m, n}$ (see (5)), we obtain the assertion of the lemma.

Definition. By a $(\lambda, \mu)$-condition for a sequence $\left\{r_{1}, \ldots, r_{n}\right\}$ of cyclically uncancellable words in $m$ variables we mean that the following restrictions hold:

(a) the sequence $\left\{r_{1}, \ldots, r_{n}\right\}$ satisfies condition $C^{\prime}(\lambda)$;

(b) among the words $r_{1}, \ldots, r_{n}$ there are no true powers;

(c) if $w$ is a cyclic subword of some $r_{i}, 1 \leq i \leq n$, and if $|w| \geq\left|r_{i}\right| / 2$, then $w$ is not $\mu$-readable.

The number of cyclically uncancellable words $r_{i}$ of length $\leq t$ that have the form $s^{d}$ is at most $(2 m-1)^{t / d} \leq(2 m-1)^{t / 2}$ for $d \geq 2$. Summing over all $d=2, \ldots, t$ and $i=1, \ldots, n$ and taking into account the bound (5), we see that the number of all sequences $\left\{r_{1}, \ldots, r_{n}\right\}$ of cyclically uncancellable words of length $\leq t$ for which condition (b) fails is less than

$$
n t(2 m-1)^{(n-1 / 2) t} \text {. }
$$

Lemma 4. If $\mu$ satisfies inequality (4), then the fraction of the sequences $\left\{r_{1}, \ldots, r_{n}\right\}$ of cyclically reduced words in the alphabet $X_{m}$ satisfying the $(\lambda, \mu)$-condition among all $n$-term sequences of words of length $\leq t$ is at least $1-\exp (-\alpha t)$ for all $t>T=T(\mu, \lambda, m, n)$ for some $\alpha=\alpha(\mu, \lambda, m, n)>0$.

Proof. Let us estimate the number of sequences for which item (c) of the $(\lambda, \mu)$-condition for $i=1$ fails. By Lemma 2, the number of these words $r_{1}$ is less than

$$
\sum_{1 \leqslant d \leqslant t} d \sum_{d / 2 \leqslant \ell \leqslant d} k\left(2 m-\frac{3}{2}\right)^{\ell}(2 m-1)^{d-\ell}<2 k t^{2}\left(2 m-\frac{5}{4}\right)^{t} .
$$

Hence, the number of sequences $\left\{r_{1}, \ldots, r_{n}\right\}$ in which $r_{1}$ does not satisfy item (c) is less than

$$
2 k t^{2}(2 m-5 / 4)^{t}(2 m-1)^{t(n-1)} \text {. }
$$

This, together with the assertion of Lemma 3 and bounds (5) and (6), implies the lemma. 


\section{§4. Subgroups with small number of generators}

In the proof of the theorems, by Lemma 4, it suffices to show that the subgroups with $\leq(m-$ 1) generators in an arbitrary group $G$ that satisfies the $(\lambda, \mu)$-condition for some $\lambda$ and $\mu$ verifying condition (4) are free. (For $t \leq T$ it simply suffices to choose a sufficiently small positive parameter $c=c(m, n)$ and take empty words $r_{1}, \ldots, r_{n}$ in (1), because we have $N_{m, n, t}^{f} \geq 1$.) Now we assume that

$$
\lambda \leq \frac{\mu}{15 m+3 \mu}<\frac{1}{6} .
$$

We shall argue by contradiction. Consider a subgroup $H$ of $G$ such that the minimal number $s$ of generators of $H$ is $\leq m-1$. In this case the marked graph $\Gamma$ that defines the subgroup $H$ has the Euler characteristic $>1-m$. Assume that we choose a graph $\Gamma$ with minimal possible number of edges. In particular, this graph is reduced.

Assume that $H$ is not free. Then the kernel of the natural homomorphism $\pi_{1}(\Gamma) \rightarrow H$ contains a nontrivial element, i.e., $\Gamma$ admits a cycle $p$ whose mark is a word that is trivial in $G$. Moreover, the path $p$ is reduced, i.e., it contains no subpaths of the form $e e^{-1}$. Since the graph $\Gamma$ is reduced, the word $w \equiv \varphi(p)$ is also reduced.

By the Grindlinger lemma for $C^{\prime}(\lambda)$-groups with $\lambda \leq 1 / 6$ (see [4]), the word $w$ contains a subword $v$ that is also a subword of a cyclic permutation $r$ of some defining word $r_{i}^{ \pm 1}$, and we have $|v|>(1-3 \lambda)\left|r_{i}\right|$.

Let $p=p_{1} \ldots p_{t}$ be a partition of the path $p$ into maximal arcs, and let the word $v$ be readable along the arcs $\bar{p}_{k}, \bar{p}_{k+1}, \ldots, \bar{p}_{\ell}$, i.e.,

$$
v \equiv v_{k} v_{k+1} \ldots v_{\ell}, \quad \text { where } \varphi\left(\bar{p}_{k+1}\right) \equiv v_{k+1}, \ldots, \varphi\left(\bar{p}_{\ell-1}\right) \equiv v_{\ell-1}, \quad \varphi\left(\bar{p}_{k}\right) \equiv v_{k}, \quad \text { and } \quad \varphi\left(\bar{p}_{\ell}\right) \equiv v_{\ell},
$$

where $\bar{p}_{k}$ is the endpoint of the arc $p_{k}$, while $\bar{p}_{\ell}$ is the starting point of the arc $p_{\ell}$, and $\tilde{p}_{j}=p_{j}$ for $j=k+1, \ldots, \ell-1$.

Let us consider several possibilities.

1. Among the words $v_{k}, v_{k+1}, \ldots, v_{\ell}$, there is a word $v_{j}$ such that $\left|v_{j}\right| \geq 5 \lambda|r|$.

By conditions (a) and (b), the arc $p_{j}^{ \pm 1}$ cannot occur twice in the sequence $p_{k+1}, \ldots, p_{\ell-1}$. The same conditions mean that if $j \neq k, \ell$ and if $p_{j}^{ \pm 1}$ occurs twice in the sequence $p_{k}, \ldots, p_{\ell}$, say, $p_{j_{0}}=p_{j}$ for $j_{0} \neq j$, then $j_{0}=k$ (or $j_{0}=\ell$ ), and in this case we have $\left|v_{k}\right|<\lambda|r|\left(\left|v_{\ell}\right|<\lambda|r|\right.$, respectively).

If $p_{3}^{ \pm 1}$ occurs only once among $p_{k}, \ldots, p_{\ell}$, then we can use a transformation of the third kind. First we join the vertices $\left(\bar{p}_{k}\right)_{-}$and $\left(\bar{p}_{\ell}\right)_{+}$by an arc of length $|r|-|v|$ with mark $u$ such that $r \equiv v u^{-1}$, and then delete an arc $p_{j}$ from $\Gamma$ (we use the relation $\varphi(u)=\varphi(v)$ in $G$ ). Then the number of edges in $\Gamma$ decreases because $|r|-|v|<3 \lambda|r|<\left|p_{j}\right|$, and the Euler characteristic is preserved. We obtain a contradiction to the minimality condition in the choice of $\Gamma$.

In the second version, we join the vertices $\left(\bar{p}_{k}\right)_{+}$and $\left(\bar{p}_{\ell}\right)_{+}$for the case $\left|v_{k}\right|<\lambda|r|$ by an arc. The mark of this arc has the length $|r|-|v|+\left|v_{k}\right|<4 \lambda|r|$ (or the length $|r|-|v|+\left|v_{k}\right|+\left|v_{\ell}\right|<5 \lambda|r|$ if we additionally have $\left|v_{\ell}\right|<\lambda|r|$ ), and this mark is equal in $G$ to an element represented by the word $v_{k+1} \ldots v_{\ell}$. Therefore, now by deleting the arc $p_{j}$, we obtain a contradiction as above.

Consider also the case $p_{k}=p_{\ell}^{ \pm 1}$ and $\left|v_{k}\right| \geq 5 \lambda|r|$. As above, for $p_{k}=p_{\ell}^{-1}$ we have $\left|v_{\ell}\right|<\lambda|r|$ by the condition $C^{\prime}(\lambda)$. However, if $p_{k}=p_{\ell}$, then in the decomposition $p_{k}=q^{\prime} q$, where $|q|=\left|v_{k}\right|-[\lambda|r|]$, the edges of the arc $q$ do not occur in the subpath of the path $p_{\ell}$ on which the word $v_{\ell}$ is written. In this case a contradiction is obtained by deleting the arc $q$ (and adding a new arc of length $<4 \lambda|r|$ ).

2. $\left|v_{j}\right|<5 \lambda|r|$ for $j=k, \ldots, \ell$.

In this case we first note that since the graph $\Gamma$ is reduced, it follows from restrictions on the Euler characteristic that the number of different maximal arcs in $\Gamma$ is at most $3 m-1$. Therefore, the word $v$ can be read on a connected subgraph of the graph $\Gamma$ with Euler characteristic $\geq 1-m$ and the number of edges $\leq 3 m 5 \lambda|r| \leq \mu(1-3 \lambda)|r|<\mu|v|$ by the choice of $\mu$ and $\lambda$. Since $v$ is a subword of $r$ of length $>|r| / 2$, we obtain a contradiction to the $(\lambda, \mu)$ condition. To complete the proof, it suffices to find a free subgroup of rank 2 in any group of the class in question. Here we can assume in addition that $\left|r_{z}\right| \geq 12$ for $i=1, \ldots, n$. (This condition fails for finite sequences whose number is exponentially small in comparison with $N_{m, n, t}$, because it is less than $\left.n(2 m-1)^{(n-1) t+11}.\right)$ 
Let us specify the number $d$ by setting $d=\max _{1 \leq i \leq n}\left|r_{i}\right|$ and verify that $x_{1}^{d}$ and $x_{2}^{d}$ generate a free subgroup. If we assume that $w\left(x_{1}^{d}, x_{2}^{d}\right)=1$ in $G$ for a nonempty cyclically uncancellable word $w$, then it follows from the Grindlinger lemma cited above that in some word $r_{i}^{ \pm 1}$ there is a cyclic subword $v \equiv x_{j}^{\ell}$, where $j=1$ or $j=2$ and $\ell>\left|r_{i}\right| / 4$. However, for the case $\left|r_{i}\right| \geq 12$, this contradicts conditions (a), (b), and (7), because two different cyclic occurrences of the word $x_{j}^{\ell-1}$ in $r_{i}^{ \pm 1}$ are of length $\geq\left|r_{i}\right| / 6$.

We can readily see that in the proof of Theorem 2 we can neglect the sequences $\left\{r_{1}, \ldots, r_{n}\right\}$ such that $\left|r_{1}\right|+\cdots+\left|r_{n}\right|=d$, but we have $\left|r_{i}\right|<\sqrt{d}$ for at least one $i \leq n$. However, if we assume that $\left|r_{i}\right|=d_{i} \geq \sqrt{d}$ for all $i$, then for all ratios $N_{d_{1} \ldots d_{n}}^{f} / N_{d_{1} \ldots d_{n}}$ we obtain estimates that are uniformly close to 1 (i.e., do not depend on the choice of the numbers $d_{1} \ldots d_{n}$ such that $d_{1}+\cdots+d_{n}=d$ ) in the same way as in above Lemmas 3 and 4 . To complete the proof of Theorem 2, we can choose the parameters $\mu$ and $\lambda$ as in Theorem 1.

In a subsequent paper, we intend to get rid of the restriction " $<m$ " on the number of generators and replace it by an arbitrarily given bound " $\angle L$ " (possibly $L \gg m$ ) by proving that the property of being free is generic for all $\leq L$-generated subgroups of infinite index in the group $G$.

The research of the second author was partially supported by the Russian Foundation for Basic Research under grant No. 94-0101541 and by the International Science Foundation under grant No. MID000.

\section{References}

1. V. S. Guba, "Conditions under which 2-generated groups in small cancellation groups are free," Izv. Vyssh. Uchebn. Zaved. Mat., [Soviet Math. J. (Iz. VUZ)], No. 7, 12-19 (1986).

2. Kourov Notebook. (Unsolved Problems of Group Theory) [in Russian], 11th ed., Nauka, Novosibirsk (1990).

3. S. W. Margolis and J. C. Meakin, "Free inverse monoids and graph immersions," Int. J. Algebra and Comput. 3, No. 1, 79-100 (1993).

4. Roger C. Lyndon and Paul E. Shupp, Combinatorial Group Theory, Springer-Verlag, Berlin-Heidelberg-New York (1977).

(G. N. Arzhantseva) Moscow State University

(A. YU. Ol'shanskit) Moscow State University

E-mail address: olsh@nw.math.msu.su

Translated by A. I. Shtern 